\title{
Penetrating Brain Injury : Wooden Stick
}

\author{
Rais Fadhlan Siregarl, Mahyudanil'2, Ridha Dharmajaya ${ }^{3}$ \\ ${ }^{1}$ Resident of Neurosurgeon, Department of Neurosurgery,University of Sumatera Utara \\ ${ }^{2}$ Staff of Department of Neurosurgery, University of Sumatera Utara \\ ${ }^{3}$ Head of Department of Neurosurgery,University of Sumatera Utara
}

\begin{abstract}
Accidental wooden penetrating brain injury is an unusual occurrence in emergency practice. At time, it could be potentially life threatening. We report an interesting case of penetrating brain injury with a wooden stick in a 20-years-old male patient. The wooden was removed successfully through craniectomy debridement.
\end{abstract}

Keyword: Brain Injury, Penetrating Injury

\section{Introduction}

Penetrating brain injury (PBI) is a traumatic brain injury (TBI) caused by low velocity sharp objects (e.g., a knife) or high-velocity projectiles (shell fragment or bullets) ${ }^{1}$. Accidental penetrating brain injury is relatively uncommon representing about $0.4 \%$ of all head injuries. Penetrating brain injury (PBI) were divided into Missile and Non missile penetrating head injuries (NPHIs) ${ }^{2}$. Non missile penetrating head injuries (NPHIs) are relatively rare compared with missile injuries. However, reports of NPHIs are increasing worldwide. First case was described in the literature as early as 1806, many intracranially penetrated foreign objects with various causes have been described. As there is significant lack of data pertaining to the management of penetrating brain damage by foreign body, it is pertinent to understand the mechanism, anatomy, and treatment of such injuries ${ }^{1}$.

\section{Case Report}

A 20-year-old male presented to our emergency department with Decreased of Conciousness. History revealed that he was riding motorcycle and had an crash injury with other motorcycle rider head to head. He fell down and a broken wooden piece entered into his skull. On physical examination, Airway was clear, Breathing was spontan with respiratory rate of 26 times per minutes, Circulatory finding were warm skin, pulse rate of 118 per minute and blood pressure

*Corresponding author at: raisfadhlan@yahoo.com

E-mail address: raisfadhlan@yahoo.com 
of 140/80 mm Hg. Glasgow Coma Scale (GCS) score on admission was E3M5V4. Pupils were unequal, Left pupil was normal in size and reacting to light, whereas right pupil was dilated and not reacting to light. There was a sutured wound over Left Frontal. Rest of the neurological examination findings were within normal limits. Patient was referred from rural Hospital.

Initial computerized tomography (CT) scan of the head shows Foreign Bodies [Figure 1]. The foreign body sites on Left frontal lobe. From bone window shows an open fracture on the Left Frontal of Skull [Figure 2].

Then we performed craniectomy debridement to clean a wound and remove all foreign bodies. A wooden penetrating bodies was pull out, then we rinsed a wound. We needed 4 burrhole in operation procedure, then performed cranicetomy debridement. The most concern we had on operation was the foreign body penetrated on the region near the anterior cerebral artery. The removal of the foreign object didn't cause any significant bleeding thus, we may assumed that it didn't penetrated any major blood vessel.

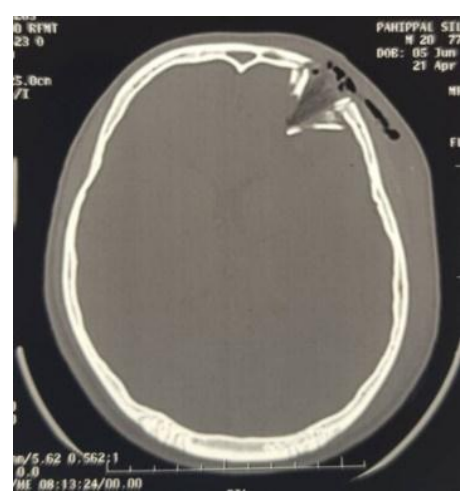

[ Figure 1]

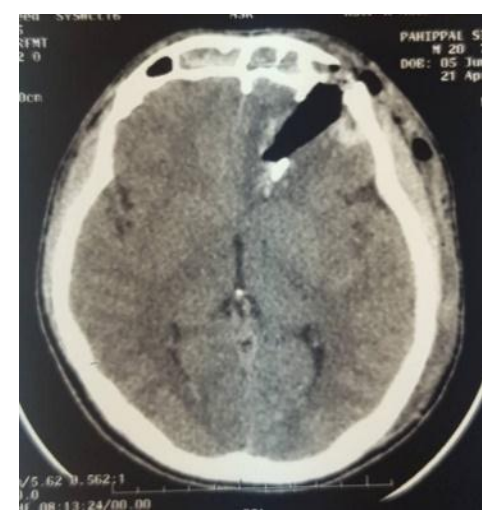

[ Figure 2]

Figure 3 showed part of penetrating wooden into brain tissues. Figure 4 shows a craniotomy debridement wound and removal wooden foreign bodies.After removal was showen in Figure 5

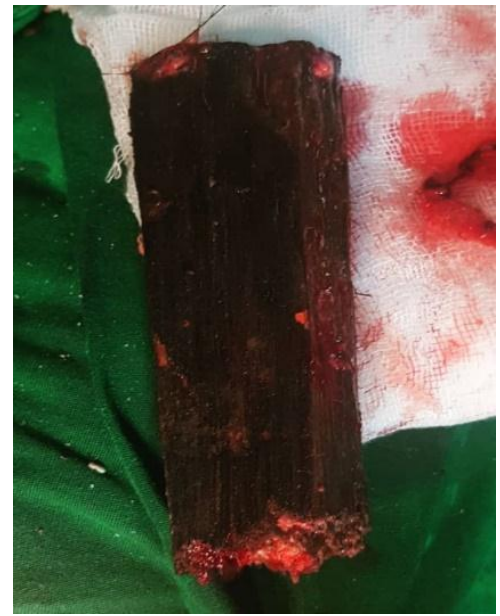

[ Figure 3]

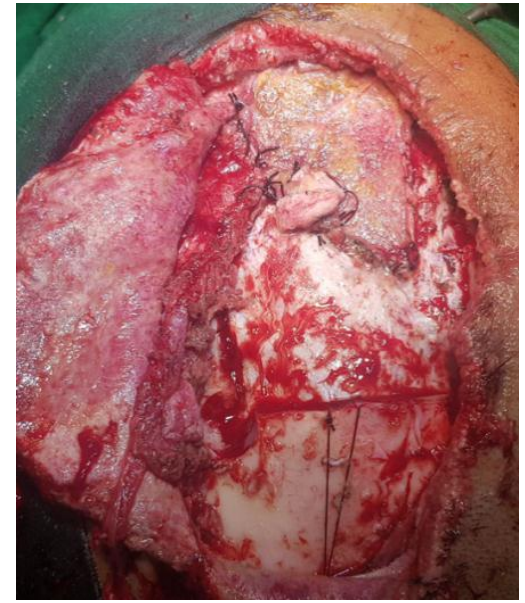

[ Figure 4]

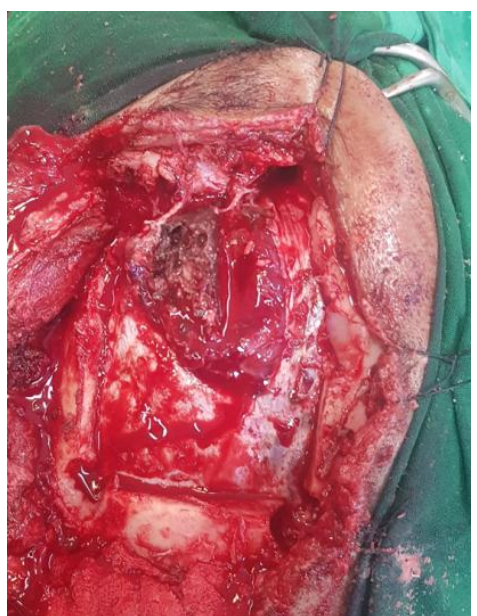

[ Figure 5] 
Figure 6 and 7 showed post operating non Contrast CT scan of the Head after 7 days in ward. Patient was treated with triple antibiotic regiment and was discharged on 7th day post operation. The recovery of the patient went uneventfull. Patient gained back his conciousness without any neurological deficit.

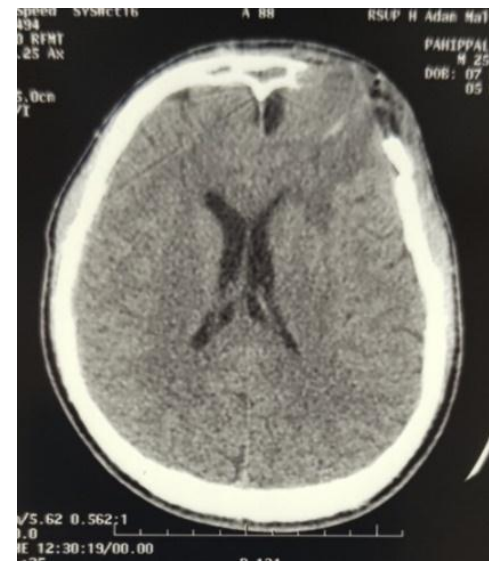

[ Figure 6]

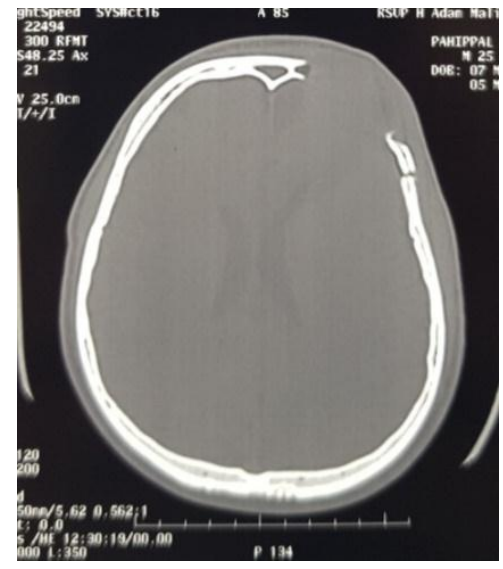

[ Figure 7]

\section{Discussion}

Accidental penetrating brain injury is relatively uncommon representing about $0.4 \%$ of all head injuries. This case report is one of non missile penetrating head injury by wooden foreign bodies. Non missile penetrating head injuries (NPHIs) are relatively rare compared with missile injuries $^{2}$. The pathophysiology is similar to closed head injuries causing cerebral contusion or intracranial hemorrhage, but there is more likelihood chance of infection. Direct crushing and laceration along the tract of penetrating injury is the initial event. As in closed head injury, intracranial pressure is likely to increase due to swelling or bleeding due to crushing of brain tissue, but most deaths are caused by damage to blood vessels which can lead to hemorrhage and ischemia ${ }^{3}$.

Immediately after arrival of the patient in the emergency department, a primary survey and stabilization of the patient with regard to the airway, breathing, cervical spine, and circulation including external hemorrhage should be achieved. After resuscitation, an inspection of the superficial wound should be done. The skin, especially the scalp, must be examined meticulously for wounds as it may be covered by blood-matted hair.An entrance wound should be identified and its location recorded as well as any exit wounds when they exist. Any CSF, bleeding, or brain parenchyma oozing from the wound should be noted; the size of the deficit should also be documented. To examine the head and neck thoroughly, the cervical collar should be removed, but strict spine precautions must be employed. Hemotympanum suggests a skull base fracture. All orifices must be checked for retention of foreign bodies, the missile, teeth, and bone. ${ }^{3,4}$ 
After careful examination of the wound, a detailed neurological examination should be performed, and the post-resuscitative glasgow coma scale (GCS) of the patients should be documented. Clinical features suggestive of raised intracranial pressure (ICP) must be documented carefully. Neurological examination, which should be followed by a complete examination of other organ systems, is recommended as PBI patients may have multiple organ injuries $^{3}$. A detailed medical history from family or friends and a chronology of the incidence from a witness is warranted. Initial laboratory evaluation must include an arterial blood gas, electrolytes, complete blood count, coagulation profile, type and cross match, and an alcohol and drug screen. Once the initial evaluation is done, the patient should be transferred to radiology for a neuroimaging. ${ }^{2,4}$

The utility of various neuroimaging methods used in patients with PBI lies on the potential management and prognostic implications of these modalities. Important findings include: entry and exit sites; intracranial fragments; missile track and its relationship to both blood vessels and air-containing skull-base structures; intracranial air; transventricular injury; basal ganglia and brain stem injury; missile track crossing the midline; multi-lobar injury; basal cisterns effacement; brain parenchymal herniation and associated mass effect. Neuroimaging is vital for surgical decision making, the type of surgery, the size and site of craniotomy, the route for extraction of foreign body, etc. as well as the decision to choose non-surgical management, which is also not uncommon in PBI. ${ }^{4}$

Plain radiographs of the skull can be of considerable value in identifying the cranial wound(s), the location of missile and bone fragments, and the presence of intracranial air. However, evaluating the projectile trajectory with plain radiographs alone can be misleading in the presence of intracranial ricocheting or fragmentation. Besides, the availability of computed tomography (CT) scanning largely precludes the use of plain radiography, and it is not routinely recommended ${ }^{3}$.

Computed tomography (CT) scanning of the head is now the primary modality used in the neuroradiologic evaluation of patients with PBI. This is because CT scanning is quick and provides improved identification of in-driven bone and missile fragments, characterization of the missile trajectory, evaluation of the extent of brain injury, and detection of intracranial hematomas and mass effects. All patients with craniocerebral gunshot injury should be imaged emergently with unenhanced CT regardless of whether or not there is evidence of penetration on clinical examination. In addition to the standard axial views with bone and soft tissue windows, coronal and sagittal sections are also helpful for patients with skull base involvement or high convexity injuries ${ }^{3}$. Postoperatively, CT scan can be helpful in evaluating the development of intracranial hematomas, the presence of residual foreign body, and the extent of cerebral edema. 
Magnetic resonance imaging (MRI) is generally not recommended for use in the acute management of PBI, as it is time consuming and can be potentially dangerous when there are retained ferromagnetic objects because of possible movement of the object in response to the magnetic torque. However, MRI can be a useful neuroradiologic modality, if the PBI is caused by a wooden object. ${ }^{3,4}$

Therapy of Penetrating Brain Injury was depends on kinds of wounds. Treatment of small entrance bullet wounds to the head with local wound care and closure in patients whose scalp is not devitalized and have no significant intracranial pathologic findings is recommended in the Guidelines for the Management of Penetrating Brain Injury ${ }^{3}$. More extensive wounds with nonviable scalp, bone, or dura should be debrided more extensively before primary closure or grafting to secure a watertight wound. When there is significant mass effect, necrotic brain tissue should be debrided and safely accessible fragments should be removed. Intracranial hematomas with significant mass effect should be evacuated. Routine surgical removal of bone or missile fragments lodged distant from the entry site especially in the eloquent areas of the brain is not recommended. Although removal of these foreign bodies from eloquent cortex may decrease the risk of posttraumatic epilepsy it has been documented to correlate with worse outcomes and higher morbidity. A conservative approach toward cerebral debridement in general has been recommended. ${ }^{4}$

Surgical treatment should be performed within $12 \mathrm{~h}$ of the injury to decrease the risk of infectious complications. Surgical incision should be done in such a fashion so as to incorporate (if possible) the area that needs debridement and vascular supply of the flap ${ }^{3}$. When the trajectory of the missile violates an open air sinus, a water tight closure of the dura should be done as the literature suggests that it may decrease the risk of abscess formation and CSF fistula. $^{4}$

\section{Conclusion}

The management of PBI differs considerably from nonpenetrating brain injury because of the unique mechanism of injury and pathophysiology involved in this type of trauma. The Guidelines for the Management of Penetrating Brain Injury has revolutionized the medical and surgical management of PBI during the last decade. There has been a paradigm shift toward a less aggressive debridement of deep seated fragments and a more aggressive antibiotic prophylaxis in an effort to improve outcomes. However, there is still a need for large scale multicenter randomized controlled trials to evaluate the current guidelines. Research in this area is highly warranted as PBI patients still present a significant challenge to practicing neurosurgeons worldwide ${ }^{3}$. 


\section{REFERENCES}

[1] Sanjay Kumar Bahera, S. P. (2016). Transorbital Penetrating Brain Injury by Wooden Stick : An Ophthalmologic Neuroemergeny. NCBI, 321-322.

[2] Xi-Sheng Li, J. Y.-S.-w. (2017). Nonmissile Penetrating Head Injury: Surgical Management and Review Literature. www.WORLDNEUROSURGERY.org, 873.e9873.e25.

[3] Syed Faraz Kazim, M. S. (2011). Management of penetrating brain injury. J Emerg Trauma Shock, 392-402

[4] H, R. W. (2016). Youmanns And Winn : Neurological Surgery, Seventh Edition. New York: Elsevier. 